\title{
Evapotranspiração e coeficientes de cultivo simples e dual do coentro
}

\author{
Vicente de PR da Silva ${ }^{1}$; Alexandra L Tavares ${ }^{1}$; Inajá Francisco de Sousa ${ }^{2}$ \\ ${ }^{1}$ UFCG-UACA, 58429-900 Campina Grande-PB; vicente@dca.ufcg.edu.br; ${ }^{2}$ UFS-DEA, Av. Mal. Rondon s/n, Rosa Elze, $49100-000$ São \\ Cristovão-SE; inajafrancisco@gmail.com
}

\begin{abstract}
RESUMO
O estudo foi conduzido na Unidade de Produção de Hortaliças localizada no município de Itabaiana-SE, com a cultura de coentro, de 2 de agosto a 12 de outubro de 2011. O objetivo do trabalho foi determinar a evapotranspiração e os coeficientes de cultivo simples e dual do coentro cultivada em climas tropicais. O coeficiente de cultivo dual foi obtido de acordo com a metodologia da FAO-56. A evapotranspiração da cultura foi obtida pelo método do balanço hídrico no solo e a evapotranspiração de referência pelo método de Penman-Monteith, utilizando-se os dados coletados numa estação automática localizada próxima à área experimental. Os valores do coeficiente de cultivo do coentro nos estádios fenológicos inicial, desenvolvimento, médio e final foram, respectivamente, 0,$82 ; 1,03$; 1,07 e 0,93 . Os resultados obtidos permitiram constatar que a metodologia do Kc dual pode ser utilizada com precisão na estimativa das necessidades hídricas da cultura do coentro em regiões tropicais. Os valores das necessidades hídricas do coentro pelo método do balanço hídrico do solo e pela metodologia do Kc dual são, respectivamente, 332 e $348,7 \mathrm{~mm}$.
\end{abstract}

Palavras chave: Coriandrum sativum, balanço hídrico no solo, necessidades hídricas, umidade do solo.

\begin{abstract}
Evapotranspiration and simple and dual crop coefficients of coriander

The study was carried out in a vegetable production unit in Itabaiana, Sergipe state, Brazil, with coriander crop from August $2^{\text {nd }}$ to October $12^{\text {th }}, 2011$. The objective of this study was to determine the evapotranspiration and single and dual crop coefficients of coriander grown in tropical environment. The dual coefficient was obtained following FAO nomenclature. The crop evapotranspiration was obtained based on soil water balance and reference evapotranspiration by Penman-Monteith method (FAO-56), using the data of air temperature, relative humidity, wind speed and solar radiation from automatic weather station next to the experimental site. The coriander crop coefficient values for the growth stages: initial, development, mid-season and late season are, respectively, 0.82; 1.03; 1.07 and 0.93 . Results also indicated that the dual crop coefficient approach can provide accurate estimates of water requirements for coriander growth in tropical environment. The water requirements of coriander based on soil water balance and the dual crop coefficient method are, respectively, 332 and $348.7 \mathrm{~mm}$.
\end{abstract}

Keywords: Coriandrum sativum, water balance, water requirement, soil water content.

(Recebido para publicação em 21 de maio de 2012; aceito em 19 de abril de 2013) (Received on May 21, 2012; accepted on April 19, 2013)

\begin{abstract}
A demanda crescente pelos recursos hídricos para atender ao consumo humano, bem como a indústria e a agricultura irrigada, têm estimulado a pesquisa e a adoção de práticas que visam aperfeiçoar o seu uso. Dentre essas demandas se destacam aquelas relacionadas com o estudo das necessidades hídricas das culturas, em que se procura identificar as exigências das plantas em seus diferentes estádios de desenvolvimento (Silva et al., 2009). Outro fator também relevante neste contexto é a determinação do coeficiente de cultura $(\mathrm{Kc})$, que se caracteriza como componente importante no cálculo de estimativas das necessidades hídricas de culturas específicas às condições edafoclimáticas do local onde a cultura se desenvolve.

$\mathrm{O}$ conhecimento dos valores preci-
\end{abstract}

sos do Kc é particularmente importante para a determinação das necessidades hídricas das culturas, tanto em termos de manejo de água na irrigação, como também no planejamento de sistemas hidroagrícolas. Ressalta-se que o uso inadequado dos métodos de estimativa da evapotranspiração de referência (ETo) às condições climáticas, a falta de precisão na estimativa, bem como o erro devido ao uso de instrumentos de medições, em geral, conduzem ao manejo inadequado da água, afetando muitas vezes a produção agrícola (Silva, 2004; Silva et al., 2005). Nesse particular, muitas pesquisas já foram realizadas visando a determinação do consumo hídrico de diferentes culturas no Brasil (Silva et al., 2001; Azevedo et al., 2003; Azevedo et al., 2006; Azevedo et al., 2008; Souza et al., 2008; Silva et al., 2009). Entretanto, nenhuma delas trata do coeficiente de cultivo dual, em especial para hortaliças, foco principal deste trabalho.

A relação dos conceitos da evapotranspiração de referência (ETo) e do Kc dual é considerada a abordagem mais utilizada em termos de acurácia dos valores de evapotranspiração (Allen et al., 1998). A metodologia do Kc dual consiste na conjunção do coeficiente basal da cultura $(\mathrm{Kcb})$ e do coeficiente de evaporação do solo - Ke (Allen, 2000). Sendo a demanda atmosférica representada pela ETo, a variação das características específicas da cultura se dá através do $\mathrm{Kc}$, com o estágio fenológico, a umidade do solo de uma forma limitada e com o tempo (Allen et al., 1998). Para a aquisição do Ke é importante o cálculo do balanço hídrico 
diário do solo, nos quais são necessários como variáveis de entrada parâmetros físicos do solo, como capacidade de campo, ponto de murcha permanente e água evaporável. Assim, tendo em vista a importância da agricultura para a economia brasileira, experimentos agrometeorológicos específicos para a determinação do Kc dessas culturas irão propiciar informações relevantes para a agricultura irrigada.

O coentro é uma hortaliça muito pouco estudada, apesar de ser considerada no ramo alimentício rica em vitaminas e sais minerais e com capacidade de produzir grandes quantidades de sua planta por unidade de área. As condições edafoclimáticas brasileiras permitem cultivar várias espécies desses vegetais durante o ano todo com o uso de irrigação. Essa olerícola é consumida em quase todo mundo, em face de suas excelentes características nutritivas na culinária, alto teor de vitaminas, propriedades medicinais, e considerável valor e importância sócio-econômica mundial. Diante disso, a demanda pela busca do aumento da produção em relação à olericultura é cada vez mais exigida, tanto no ramo da saúde quanto no agronegócio. Ela é uma hortaliça herbácea anual pertencente à família Apiaceae, nativa da bacia do Mar Mediterrâneo. A sua versatilidade de uso propicia a essa olerácea grande valor e importância sócio-econômica mundial, destacando-se mais na Ásia, onde se localizam os países líderes de produção e consumo, com grande importância para a culinária, utilizações aromáticas e medicinais (Diederichsen, 1996; Ramadan \& Wahdan, 2012), e, também, com ampla utilização na indústria alimentícia (Aluko et al., 2001; Burdok \& Carabin, 2009; Michalczyk et al., 2012), farmacêutica (Jabeen et al., 2009) e cosméticos (Eyres et al., 2005). Considerando a importância dessa cultura e a ausência de informação sobre o seu manejo hídrico, o presente trabalho tem o objetivo de determinar as necessidades hídricas e o coeficiente de cultivo da cultura de coentro nas condições edafoclimáticas brasileiras.

\section{MATERIAL E MÉTODOS}

O presente estudo foi conduzido na
Unidade de Produção de Hortaliças, em área do "Projeto Pequeno Produtor Grande Empreendedor", parceria entre a Universidade Federal de Sergipe e Grupo G. Barbosa, no município de Itabaiana-SE $\left(10^{\circ} 41^{\prime} 06^{\prime} \mathrm{S}, 37^{\circ} 25^{\prime} 31^{\prime \prime} \mathrm{O}\right.$, altitude $188 \mathrm{~m})$, centrado na faixa centro-ocidental do estado de Sergipe.

O solo da área experimental é classificado como Podzólico Vermelho-Amarelo (povoado Água Branca), de textura areno-argilosa e de média fertilidade (Nunes, 2002). A análise física do solo da área experimental indicou textura arenosa, sendo $80 \%$ areia, $13 \%$ silte e $7 \%$ argila.

A área experimental foi subdividida em esquema de sete leiras medindo 9,5 $\mathrm{m}$ de comprimento por $0,7 \mathrm{~m}$ de largura, com espaçamento entre as covas de 20 $\mathrm{cm}$. O coentro (Coriandrum sativum) utilizado no experimento foi a cultivar Verdão. A semeadura, a partir de sementes da marca Sakata, foi realizada no dia 2 de agosto de 2011, utilizando-se 4 sementes por cova, de modo a minimizar o insucesso da germinação da planta. Foram realizados os devidos tratos culturais, com as limpezas dos canteiros efetuadas no dia 25 de agosto de 2011 e incorporado ao solo $70 \mathrm{~kg}$ de esterco distribuídos nas sete leiras. Adotou-se um delineamento experimental inteiramente casualizado com sete tratamentos e quatro repetições. As leiras mediram 9,5 $\mathrm{m}$ de comprimento por $0,7 \mathrm{~m}$ de largura e a área útil foi de $80,75 \mathrm{~m}^{2}$.

O sistema de irrigação utilizado no manejo hídrico foi por micro aspersão, aplicado diariamente $\left(6,9 \mathrm{~mm} \mathrm{~d}^{-1}\right)$, nos dias que não ocorreram chuvas, de modo a suprir as necessidades hídricas da planta. Os dados observacionais para a determinação da evapotranspiração de referência foram obtidos em uma Estação Meteorológica do Perímetro Irrigado Ribeira, localizada no município de Itabaiana, localizada a cerca de 1.000 $\mathrm{m}$ da área experimental. $\mathrm{O} \mathrm{Kc}$ dual foi determinado separadamente, utilizando-se dois coeficientes: o coeficiente de cultura basal (Kcb) para descrever a transpiração da planta e o coeficiente de evaporação da água do solo (Ke) para descrever a evaporação que ocorre na superfície do solo. Este método proporciona observar mais claramente as frações da água utilizadas pela cultura, seja da precipitação ou da irrigação, de forma que o Kc seja representado conforme a equação:

$$
K_{c}=K_{c b}+K_{e}
$$

Os procedimentos para as determinações do coeficiente de cultivo basal $\left(\mathrm{K}_{\mathrm{cb}}\right)$, coeficiente de evaporação $\left(\mathrm{K}_{\mathrm{e}}\right)$ podem ser obtidos em Silva et al. (2012). A evapotranspiração do coentro (ETc) foi também determinada pelo método do balanço hídrico no solo, expresso por:

$$
E T_{c}=P+I+A-D \pm \Delta W \pm R
$$

em que Pé a precipitação pluviométrica; I a lâmina de irrigação; A a ascensão capilar; D a drenagem profunda; a variação da lâmina de água disponível no solo e R o escoamento superficial. Todos os componentes da Eq. (2) são expressos em $\mathrm{mm} \mathrm{d}^{-1}$. O escoamento superficial foi considerado nulo, visto que a topografia do terreno era plana, $\mathrm{P}$ foi adquirida junto a uma Estação Meteorológica do Perímetro Irrigado Ribeira, no município de Itabaiana, e $\Delta \mathrm{W}$ determinado com base no perfil de umidade do solo. O volume de controle considerado para elaboração do balanço hídrico corresponde à camada de solo compreendida entre a superfície e a profundidade efetiva do sistema radicular. O tamanho médio das raízes do coentro variou de $2,75 \mathrm{~cm}$ no estádio inicial a 13 $\mathrm{cm}$ no estádio médio da cultura. Como o lençol freático na área de estudo foi superior a $1 \mathrm{~m}$ de profundidade, o termo ascessão capilar foi considerado nulo e, também, como não foram instalados tensiômetros para monitorar a tensão de água no solo, a percolação foi obtida conforme procedimentos estabelecidos por Allen et al. (1998). O armazenamento de água no solo (W), em mm, durante um dado intervalo de tempo foi calculado usando-se a seguinte equação:

$$
W_{t}=\int_{0}^{\mathrm{L}} \theta(z) d z=\bar{\theta} \mathrm{L}
$$

em que $\bar{\theta}$ é a umidade do solo média até a profundidade considerada $\left(\mathrm{cm}^{3} / \mathrm{cm}^{3}\right) \mathrm{e}$ $\mathrm{L}$ a espessura da camada de solo $(\mathrm{cm})$. Durante o intervalo de tempo considerado, a variação do armazenamento de água no solo ( $\Delta \mathrm{W}$ ), expressa em $\mathrm{cm}$, foi obtida como

$$
\Delta \mathrm{W}=\mathrm{W}_{\mathrm{t}}-\mathrm{W}_{\mathrm{t}-1}
$$


em que $\mathrm{W}$ e $\mathrm{W}_{\mathrm{t}-1}$ representam os armazenamentos de água no perfil do solo nos instantes $\mathrm{t}$ e $\mathrm{t}-1$, respectivamente. A umidade do solo foi monitorada utilizando-se a sonda 5TE Decagon, modelo Em50G, instalados na área em estudo, nas profundidades 20, 40 e $60 \mathrm{~cm}$. Os valores de umidade do solo foram obtidos em intervalos horários durante o período experimental. A evapotranspiração de referência $\left(\mathrm{ET}_{\mathrm{o}}\right)$ foi obtida como descrito em Allen et al. (1998), na forma comumente designada como a equação FAO-PM.

\section{RESULTADOS E DISCUSSÃO}

Os valores dos coeficientes de cultivo e da evapotranspiração do coentro com base no balanço hídrico do solo e na metodologia do Kc dual, para todos os estádios fenológicos da cultura são exibidos na Tabela 1. De acordo com as duas metodologias, o coeficiente de cultivo e a evapotranspiração são maiores no estádio médio da cultura, enquanto nos estádios inicial e final esses parâmetros atingem valores mínimos. A metodologia do balanço hídrico no solo subestima os valores do Kc e da ET do coentro em relação à metodologia do Kc dual, muito embora com valores muito próximos.

O consumo hídrico do coentro foi de 332 e de $348,7 \mathrm{~mm}$, pelo método do balanço hídrico (BHS) e pela metodologia do coeficiente de cultivo dual (MCD), respectivamente, cuja diferença representa apenas $4,7 \%$. Este resultado é particularmente relevante haja vista a ausência na literatura corrente sobre o consumo hídrico do coentro. Por outro lado, os resultados do trabalho de Albuquerque Filho et al. (2009) com a cultura do coentro indicam que a eficiência do uso da água, o rendimento de massa verde, o rendimento de massa seca e o número de plantas, aumentaram linearmente com a elevação das doses do polímero quando se fixa a menor lâmina de irrigação.

A evapotranspiração média durante o ciclo da cultura pelo BHS foi 4,6 $\mathrm{mm}$ e pelo $\mathrm{MDC}$ foi $4,7 \mathrm{~mm}$. Por outro lado, o Kc do coentro pelo BHS variou de 0,82 (estádio inicial) a 1,07 (estádio médio) e pelo MDC variou entre 0,91

Tabela 1. Valores médios $\left(\mathrm{mm} \mathrm{d}^{-1}\right)$ e acumulados $(\mathrm{mm})$ da evapotranspiração da cultura do coentro e coeficientes de cultivos obtidos com base no balanço hídrico no solo e na metodologia do Kc dual [mean $\left(\mathrm{mm} \mathrm{d}^{-1}\right)$ and cumulative values $(\mathrm{mm})$ of crop evapotranspiration and crop coefficient of coriander based on soil water balance and dual crop coefficient method]. Itabaiana, UFS, 2011.

\begin{tabular}{lccccccc}
\hline & \multicolumn{3}{c}{ Balanço hídrico } & & \multicolumn{3}{c}{ Coeficiente de cultivo dual } \\
\cline { 2 - 3 } & Kc & $\begin{array}{c}\text { ET } \\
\text { média }\end{array}$ & $\begin{array}{c}\text { ET } \\
\text { total }\end{array}$ & & Kc & $\begin{array}{c}\text { ET } \\
\text { média }\end{array}$ & $\begin{array}{c}\text { ET } \\
\text { total }\end{array}$ \\
\hline Inicial & 0,82 & 3,9 & 70,9 & & 0,91 & 4,2 & 75,2 \\
Desenvolvimento & 1,03 & 4,7 & 112,8 & & 1,20 & 5,4 & 130,6 \\
Médio & 1,07 & 5,1 & 101,0 & & 1,22 & 5,2 & 105,0 \\
Final & 0,93 & 4,7 & 47,3 & & 0,77 & 3,8 & 37,9 \\
Total & - & & 332,0 & & - & - & 348,7 \\
Média & 0,96 & 4,6 & & & 1,02 & 4,7 & - \\
\hline
\end{tabular}

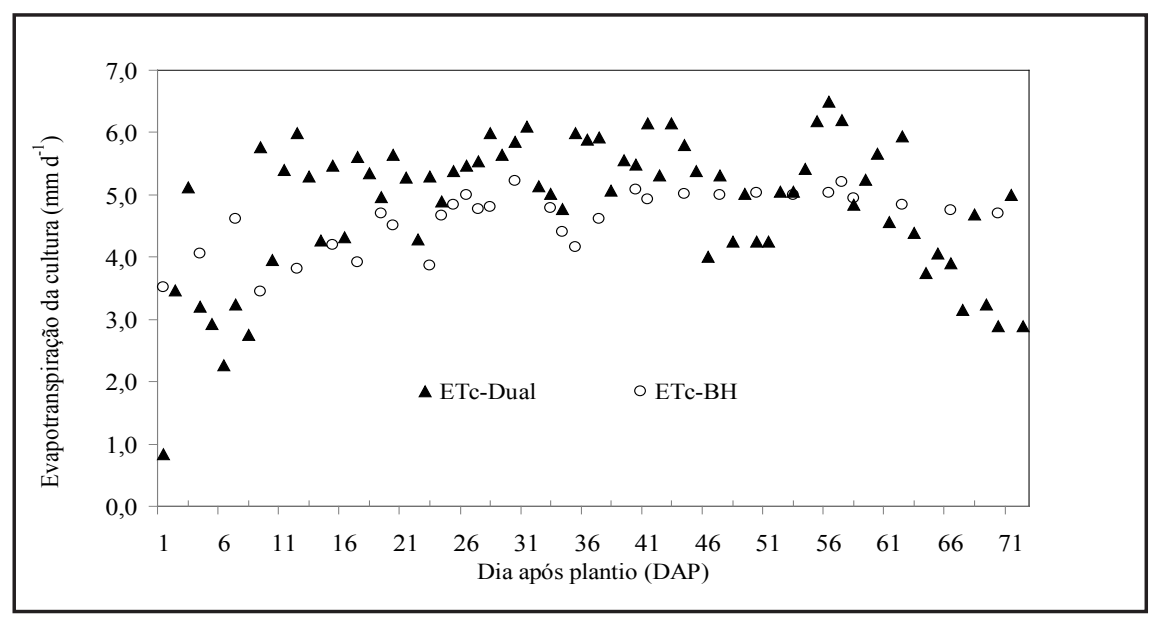

Figura 1. Curso diário da evapotranspiração do coentro pelo método do balanço hídrico no solo (ETc-BH), e pela metodologia do coeficiente de cultivo dual (ETc-Dual) durante o período experimental (daily course of evapotranspiration of coriander based on soil water balance (ETc-BH) and dual crop coefficient method [ETc-Dual]). Itabaiana, UFS, 2011.

(estádio inicial) a 1,22 (estádio médio). O estádio de desenvolvimento foi o de maior consumo hídrico de acordo com duas metodologias, sendo de $112,8 \mathrm{~mm}$ pelo BHS e de 130,6 mm pelo MDC. Esses resultados evidenciam que a metodologia do coeficiente de cultivo dual apresenta estimativas confiáveis das necessidades hídricas da cultura. Resultados semelhantes foram obtidos por Silva et al. (2012) em experimento realizado com a cultura da cana-de-açúcar nos tabuleiros costeiros do Estado da Paraíba.

O curso diário da evapotranspiração pelo método do balanço hídrico no solo e pela metodologia do coeficiente de cultivo dual durante o período experimental com a cultura do coentro em Itabaiana, no ano de 2011, é exibido na Figura 1. Como o fornecimento de água através de irrigação durante o experimento foi fixo numa taxa de $6,9 \mathrm{~mm} \mathrm{~d}^{-1}$, os valores de evapotranspiração da cultura por ambos os métodos apresentaram variações de acordo com a demanda atmosférica, expressa pela evapotranspiração de referência. Nos dias em que ocorreram os maiores índices pluviométricos, como nos DAPs 22, 25 e 48, foram registrados os menores valores de evapotranspiração, sendo, respectivamente, de 4,4; 5,7; 4,2 mm, pela metodologia do Kc dual e de 4,7; 4,8 e 4,3 mm, respectivamente, pelo método do balanço hídrico do solo. O curso diário da evapotranspiração da cultura evidencia os maiores valores nos estádios fenológicos de desenvolvimen- 


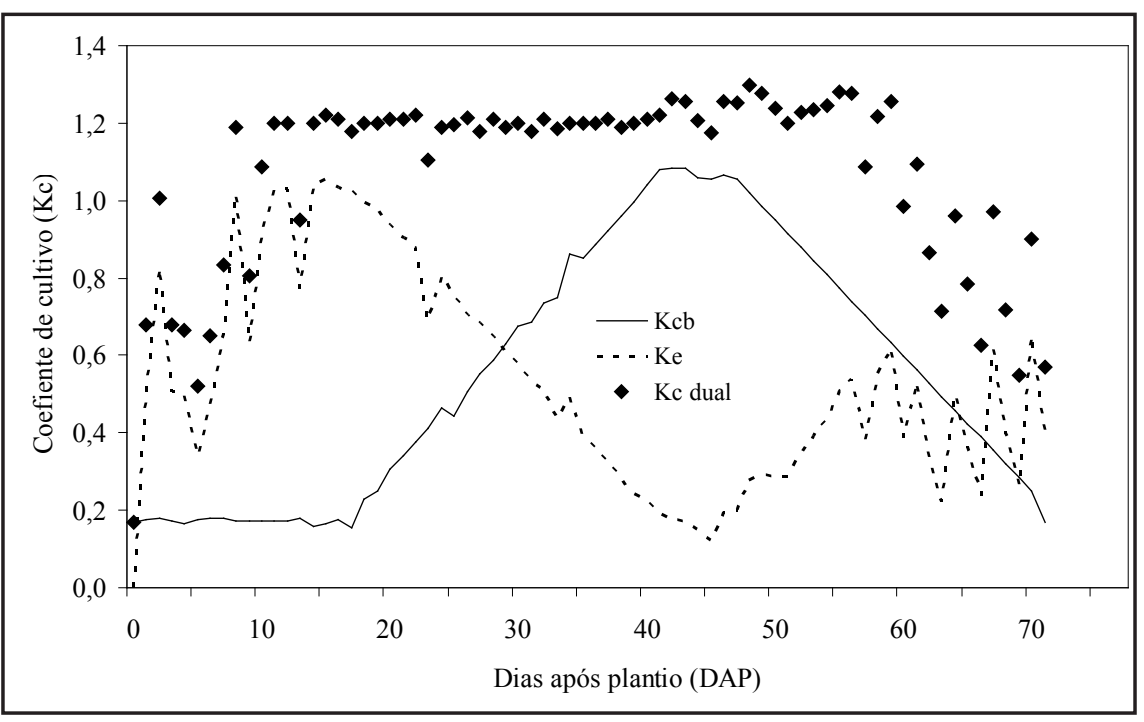

Figura 2. Curso temporal do coeficiente de cultivo basal (Kcb), coeficiente de evaporação de água do solo $(\mathrm{Ke})$ e coeficiente de cultivo dual (Kc dual) da cultura do coentro (temporal course of basal crop coefficient [Kcb], soil evaporation coefficient [Ke] and dual crop coefficient [Kc dual] for coriander crop). Itabaiana, UFS, 2011.

to e médio do coentro e os menores nos estádios inicial e final, de acordo com ambas as metodologias.

Na Figura 2 é exibido o curso temporal do coeficiente de cultivo basal, coeficiente de evaporação de água do solo e coeficiente de cultivo dual da cultura do coentro. Constata-se que a evaporação do solo $(\mathrm{Ke})$ tem um comportamento simétrico à da transpiração da planta, expressa pelo coeficiente de cultivo basal, ao longo do desenvolvimento da cultura. Isso se deve ao fato que a evaporação do solo é máxima no início do ciclo da cultura, em face do solo se encontrar descoberto pela vegetação; e, nesse caso, a transpiração é mínima. Por outro lado, à medida que a planta cresce, há o aumento de sua área foliar, e consequente aumento de sua transpiração, pois a fração de cobertura do solo passa a aumentar e a evaporação do solo passa então a diminuir, devido à redução da área do solo exposto.

A evaporação de água no solo apresenta maior variabilidade do início e final do período de cultivo; por outro lado, a transpiração, ou seja, o Kcb, manteve-se constante nos períodos inicial e médio, além de acréscimo e decréscimo lineares nos estádios de desenvolvimento e final, respectivamente. O decréscimo da transpiração no período final da cultura está associado à sua senescência que é acompanhada pelo leve acréscimo na evaporação de água no solo. Kumar et al. (2008), quando realizaram um experimento de campo em solo franco-arenoso com a cultura de coentro, em Nova Delhi, Índia, observaram que a aplicação de três níveis de irrigação nas fases de ramificação, floração e formação de sementes, produziu os maiores valores de crescimento e produção da cultura. Eles observaram ainda que a evapotranspiração da cultura foi maior com a aplicação das irrigações, mas a eficiência do uso da água manteve-se maior quando a irrigação foi suspensa na fase de formação de sementes.

No período entre os DAPs 1 e 30 é necessário um controle rigoroso da irrigação para a otimização do desenvolvimento da cultura do coentro de forma satisfatória. A metodologia do Kc dual reproduziu satisfatoriamente a variabilidade do consumo hídrico da cultura, com valores mínimos no início e final do ciclo e máximo no estádio intermediário. $\mathrm{O}$ coeficiente do estresse hídrico (Ks) foi sempre igual à unidade indicando que em face da alta lâmina de irrigação a cultura não sofreu estresse hídrico em nenhum dia do período monitorado. $\mathrm{O}$ Kcb variou do valor mínimo de 0,16 no estádio inicial, a 1,08 no estádio médio; enquanto a média \pm desvio padrão de todo o período foi de $0,55 \pm 0,33$. Os coeficientes de cultivos simples ou basal da cultura do coentro não são listados no Boletim 56 da FAO. Dessa forma, o Kcb foi determinado neste trabalho seguindo a metodologia estabelecida em tal boletim (Allen et al., 1998).

Constatou-se que os valores dos Kcb do coentro encontrados neste estudo são semelhantes àqueles sugeridos para vários tipos de pequenos vegetais. $\mathrm{A}$ característica comum desses vegetais é que o coeficiente de cultivo basal final é apenas um pouco inferior àquele do estádio médio, e às vezes até igual. Por exemplo, os valores dos Kcb da cultura do alho nos estádios inicial, médio e final são, respectivamente, 0,$15 ; 0,95$ e 0,90 ; enquanto para a cultura de alface esses valores são, respectivamente, 0,15 ; 0,90 e 0,90 (Allen et al., 1998). O coeficiente de evaporação de água no solo $(\mathrm{Ke})$ variou entre 0,1 e 1,1, com valores máximos no estádio inicial e mínimos no estádio médio. A média \pm desvio padrão do Ke durante todo o período foi de $0,5 \pm 0,3$; portanto, bastante próxima do $\mathrm{Kcb}$, porém com grande variabilidade nos estádios inicial e final da cultura. Por outro lado, o Kc dual apresentou uma variabilidade maior do que $\mathrm{Ke}$ e $\mathrm{Kcb}$, cuja média \pm desvio padrão foi de $1,1 \pm 0,2$.

Os resultados deste trabalho, que analisou o consumo hídrico do coentro com base em duas metodologias, permitem concluir os valores do coeficiente de cultivo do coentro em seus estádios fenológicos: $\mathrm{Kc}($ inicial $)=0,82 ; \mathrm{Kc}$ $($ desenvolvimento $)=1,03 ; \mathrm{Kc}$ (médio) $=1,07 \mathrm{e} \mathrm{Kc}($ final $)=0,93$. Os valores das necessidades hídricas do coentro pelo método do balanço hídrico do solo e pela metodologia do Kc dual são, respectivamente, 332 e 348,7 $\mathrm{mm}$. Em ambos os métodos, o estádio fenológico de desenvolvimento é o de maior demanda hídrica. Os resultados ainda indicaram que a metodologia do Kc-dual pode ser utilizada com precisão na estimativa das necessidades hídricas da cultura do coentro.

\section{REFERÊNCIAS}

ALBUQUERQUE FILHO JAC; LIMA VLA; MENEZES D; AZEVEDO CAV; DANTAS NETO J; SILVA JUNIOR JG. 2009. Características vegetativas do 
coentro submetido a doses do polímero hidroabsorvente e lâminas de irrigação. Revista Brasileira de Engenharia Agrícola e Ambiental, 13: 671-679.

ALLEN RG; PEREIRA LS; RAES D; SMITH M. 1998. Crop Evapotranspiration: Guidelines for Computing Crop Water Requirements. FAO Irrigation and Drainage Paper 56. Rome, 300p.

ALLEN RG. 2000. Using the FAO-56 dual crop coefficient method over an irrigated region as part of an evapotranspiration intercomparison study. Journal of Hydrology: 229: 27-41.

ALUKO RE; McINTOSH T; REANEY M. 2001. Comparative study of the emulsifying and foaming properties of defatted coriander (Coriandrum sativum) seed flour and protein concentrate. Food Research International 34: 733-738.

AZEVEDO PV; SILVA BB; SILVA VPR. 2003. Water requirements of irrigated mango orchards in northeast Brazil. Agricultural Water Management 58: 241-254.

AZEVEDO PV; SOUSA IF; SILVA BB; SILVA VPR. 2006. Water-use efficiency of dwarfgreen coconut (Cocos nucifera L.) orchards in northeast Brazil. Agricultural Water Management 84: 259-264.

AZEVEDO PV; SOARES JM; SILVA VPR; SILVA BB; NASCIMENTO T. 2008. Evapotranspiration of "Superior" grapevines under intermittent irrigation. Agricultural Water Management 95: 301-308.

BURDOK GA; CARABIN LG. 2009. Safety assessment of coriander (Coriandrum sativum L.) essential oil as a good ingredient. Food and
Chemical Toxicology 47: 22-34.

DIEDRICHSENA. 1996. Coriander (Coriandrum sativum L.). Promoting the conservation and use of underutilized and negleted crops. 3. Rome: Institute of Plant Genetics and Crop Plant Research, Gartersleben/International Plant Genetic Resources Institute. p.83.

EYRES G; DUFOUR JP; HALLIFAX G; SOTHEESWARAN S, MARRIOTT PJ. 2005. Identification of character-impact odorants in coriander and wild coriander leaves using gas chromatography-olfactometry (GCO) and comprehensive twodimensional gas chromatography-time-of-flight mass spectrometry (GOC x GC-TOFMS). Journal of Separation Science 28: 1061-1074.

JABEENQ; BASHIR S; LYOUSSIB; GILANIAH. 2009. Coriander fruit exhibits gut modulatory, blood pressure lowering and diuretic activities. Journal of Ethnopharmacology 122: 123-130.

KUMAR A; SINGH R; CHHILLAR RK. 2008. Influence of omitting irrigation and nitrogen levels on growth, yield and water use efficiency of coriander (coriandrum sativum). Acta Agronomica Hungarica 56: 69-74.

MICHALCZYK M; MACURA R; TESARPWOCZ I; BANAS J. 2012. Effect of adding essential oils of coriander (Coriandrum sativum L.) and hyssop (Hyssopus officinalis $L$.) on the shelf life of ground beef. Meat Science 90: 842-850.

NUNES MUC. 2002. Produtividade e principais problemas fitossanitários de cultivares de batata em Sergipe. Horticultura Brasileira 20: 424- 427 .
RAMADAN MF; WAHDAN KMM. 2012. Blending of corn oil with black cumin (Nigella sativa) and coriander (Coriandrum sativum) seed oils: Impact on functionality, stability and radical scavenging activity. Food Chemistry 132: 873-879.

SILVA VPR; AZEVEDO PV; SILVA BB; BASSOI LH; TEIXEIRA AHC; SOARES JM; SILVA JAM. 2001. Estimativa da evapotranspiração da mangueira com base no balanço hídrico do solo. Revista Brasileira de Engenharia Agrícola e Ambiental 5: 456-462.

SILVA VPR. 2004. On climate variability in Northeast of Brazil. Journal of Arid Environments 58: 575-596.

SILVA VPR; FILHO AFB; SILVA BB; CAMPOS JHBC. 2005. Desenvolvimento de um sistema de estimativa da evapotranspiração de referência. Revista Brasileira de Engenharia Agricola e Ambiental 9: 547-553.

SILVA VPR; CAMPOS JHBC; AZEVEDO PV. 2009. Water-use efficiency and evapotranspiration of mango orchard grown in northeastern region of Brazil. Scientia Horticulturae 102: 467-472.

SILVA VPR; BORGES CJR; FARIAS CHA; ALBUQUERQUE W; BARBOSA BB. 2012. Water requirements and single and dual crop coefficients of sugarcane grown in a tropical region, Brazil. Agricultural Sciences 3: 274286.

SOUZA CB; SILVA BB; AZEVEDO PV; SILVA VPR. 2008. Fluxos de energia e desenvolvimento da cultura do abacaxizeiro. Revista Brasileira de Engenharia Agrícola e Ambiental 12: 400-407. 